\title{
Bariatric Surgery in Prior Solid Organ Transplantation Patients: Is Race a Predictor of Adverse Outcomes?
}

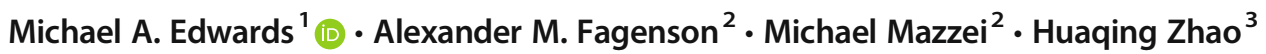

Received: 16 May 2020 / Revised: 18 June 2020 / Accepted: 22 June 2020 / Published online: 2 July 2020

(C) Springer Science+Business Media, LLC, part of Springer Nature 2020

\begin{abstract}
Purpose Metabolic and bariatric surgery (MBS) is increasingly performed in patients with previous solid organ transplantation (PSOT). In addition, controversy remains about whether racial disparity in outcomes following MBS exists. Therefore, the aim of this analysis was to determine if race independently predicts outcomes in MBS patients with PSOT.

Materials and Methods Patients with PSOT undergoing sleeve gastrectomy (SG) and Roux-en-Y gastric bypass (RYGB) were identified in the 2017 Metabolic and Bariatric Surgery Accreditation Quality and Improvement Project (MBSAQIP) database. Patients were stratified by race (Black and White). Propensity score matching was utilized to adjust for multiple demographic variables. Multivariable logistic regression analyses were performed for overall and bariatric-related morbidity.

Results Of 335 MBS patients with PSOT, $250(75 \%)$ were white and $85(25 \%)$ were black patents. Procedure-type and surgical approach $(p>0.1)$ were similarly distributed. Black patients were more likely $(p<0.05)$ to have hypertension dialysis-dependent chronic kidney disease, and be on chronic steroids). Mortality and morbidity were similar. Black patients had significantly $(p<0.05)$ higher rates of renal failure, pulmonary complications, and emergency department visits in unmatched analysis. After propensity score matching, 82 patients in each cohort were identified and were similar at baseline $(p>0.5)$. In the matched analysis, black patients had higher overall $(17 \%$ vs. $10 \%, p=0.12)$ and bariatric-related morbidity $(14 \%$ vs. $7.2 \%, p=0.05)$. In addition, black patients had significantly $(p<0.05)$ higher rates of postoperative pneumonias, progressive renal insufficiency, and emergency department visits. On multivariable regression analysis, black race did not independently predict overall or bariatric-related morbidity.

Conclusion MBS in racial cohorts with PSOT is safe, with very low rates of overall morbidity and mortality. Black race trended toward increased postoperative morbidity. Larger cohort studies are needed to validate our findings.
\end{abstract}

Keywords Bariatric surgery $\cdot$ Prior solid organ transplant $\cdot$ Outcomes $\cdot$ Racial disparity

Electronic supplementary material The online version of this article (https://doi.org/10.1007/s11695-020-04813-9) contains supplementary material, which is available to authorized users.

Michael A. Edwards

Edwards.michael@mayo.edu

1 Department of Surgery, Mayo Clinic, 4500 San Pablo Rd S, Jacksonville, FL 32224, USA

2 Department of Surgery, Temple University Hospital, Suite , 3401 North Broad Street, Philadelphia, PA 19140, USA

3 Department of Clinical Sciences, Lewis Katz School of Medicine at Temple University, Philadelphia, PA 19140, USA

\section{Introduction}

Obesity is an increasing epidemic worldwide $[1,2]$ that is associated with significant morbidity. Metabolic and bariatric surgery (MBS) has been proven as a safe and effective treatment option for severe obesity that is associated with durable weight loss, improvement and resolution of comorbid conditions [3-6], reduced cancer risk, increased longevity [7, 8], and improved quality of life $[9,10]$.

Given the increasing safety profile of MBS, there is an increasing interest in its role in higher-risk patient cohorts, including transplant candidates and patients. This increasing practice trend is driven by data showing that $2-12 \%$ of patients awaiting solid organ transplantation meet the criteria for severe obesity (body mass index $(\mathrm{BMI})>35 \mathrm{~kg} / \mathrm{m}^{2}$ ) [11] and weight loss prior to organ transplantation results in better 
perioperative outcomes and improved graft survival [12, 13]. While limited to small case series and single-institution experience, there is growing evidence suggesting that MBS can be a safe and effective weight loss treatment option in patients with previous solid organ transplant (PSOT) [11-16].

While obesity has increased among all demographics in the USA, the prevalence and health impact disproportionately impact racial and ethnic minority patients [1-3, 17-20] who continue to be an underrepresented cohort of MBS patients. Even though MBS is overall safe and effective [3-10], there remains a controversy regarding the safety profile and effectiveness of MBS surgery in racial and ethnic cohorts, with some studies reporting no outcome differences [21-23] while others report higher rates of adverse outcomes in racial and ethnic minority patients [24, 25].

No published data exists on outcomes in racial and ethnic MBS patient cohorts with PSOT. Therefore, the goal of this study goal was to compare outcomes between black and white MBS patients with a history of PSOT to determine if race was an independent predictor of perioperative outcomes.

\section{Material and Methods}

\section{Data Source}

A retrospective analysis of the 2017 Metabolic and Bariatric Surgery Accreditation and Quality Improvement Program (MBSAQIP) Participant Use Files (PUF) database was performed, and outcomes between racial cohorts of MBS patients with PSOT were compared. The MBSAQIP is responsible for the accreditation of bariatric surgical facilities. Requirements for certification includes reporting bariatric surgical outcomes to the MBSAQIP Participant Use Data File (PUF), a Health Insurance Portability and Accountability Act (HIPAA)-compliant data file registry containing prospectively entered, riskadjusted, clinically rich data using standardized definitions for preoperative, intraoperative, and postoperative variables that are specific to metabolic and bariatric surgical care. Data points are abstracted at participating institutions by certified reviewers who are audited for accuracy of performance. The 2017 file included data on a composite variable of previous solid organ transplantation (PSOT), including a history of heart, lung, liver, renal, pancreas, and bowel transplantation. This is a de-identified, nationally available, clinical database; therefore, neither institutional review board (IRB) approval nor patient consent was required for our study.

\section{Case Selection and Inclusion Criteria}

Our inclusion and exclusion criteria are detailed in Fig. 1. Patients with PSOT undergoing primary gastric bypass (RYGB) or sleeve gastrectomy (SG) cases were identified through Current Procedural Terminology (CPT) codes 43644, 43645, and 43775. Exclusions included bariatric procedure other than a RYGB or SG, bariatric procedures designated as emergency, open cases, surgical approach other than robotic-assisted or conventional laparoscopy, and other races including American Indian or Alaska Native, Asian, Native Hawaiian, or Other Pacific Islands and Unknown. Selected cases were further stratified by race (non-Hispanic black vs. non-Hispanic white).

\section{Data Collection, Matching, and Statistical Analysis}

Descriptive statistics were collected and compared between groups, including demographics, health summary status, preoperative comorbidities, and operative characteristics. Primary outcome measures included 30-day mortality and morbidity. Secondary outcome measures included other 30day adverse outcomes (reoperation, readmission, and reintervention), postoperative complication, aggregate complications (Appendix Table 7), and hospital outcomes (operative duration, conversion, and hospital length of stay). Propensity score-matched analyses were performed to adjust for intergroup biases. The matching ratio was one-to-one. A logistic regression model was generated on patient demographics and variables with a $p<0.1$ on univariate analysis between racial cohorts with prior solid organ transplantation. Variables included in our regression model were Hispanic ethnicity, hypertension (HTN), chronic steroid use, obstructive sleep apnea (OSA), dialysis-dependent chronic kidney disease $(C K D)$, and renal insufficiency. A propensity score from 0 to 1 was generated from this model and assigned to each subject. A nearest neighbor 1:1 variable ratio with propensity scores that fell within a caliper of 0.02 was then used to generate matched cohorts hypothesized to be balanced of potentially confounding baseline characteristics.

A backward method multivariable logistic regression was performed for overall and bariatric-related morbidity. Factors with a difference $(p<.05)$ on univariate analysis between the two racial cohorts were included in the model: race (Black vs. White), Hispanic ethnicity, hypertension, chronic steroid use, and dialysis-dependent CKD in our multivariate logistic regression analyses. A procedure-specific multivariable regression analysis was performed for sleeve gastrectomy and gastric bypass cases independently. For each procedure, race and variables that were significantly $(p<.05)$ on univariate analysis were included in the multivariate regression models. For SG cases, variables included in the regression algorithm were race, Hispanic ethnicity, gender, and OSA. For RYGB, variables included race, ethnicity, body mass index (BMI), and renal insufficiency.

For univariate unmatched and matched analyses, primary and secondary outcomes were compared by Pearson ChiSquare test for categorical variables and Mann-U-Whitney 
Fig. 1 Inclusion and exclusion flow diagram. MBSAQIP =

Metabolic and Bariatric Surgery Accreditation and Quality Improvement Project, BMI = body mass index, TXP = transplantation

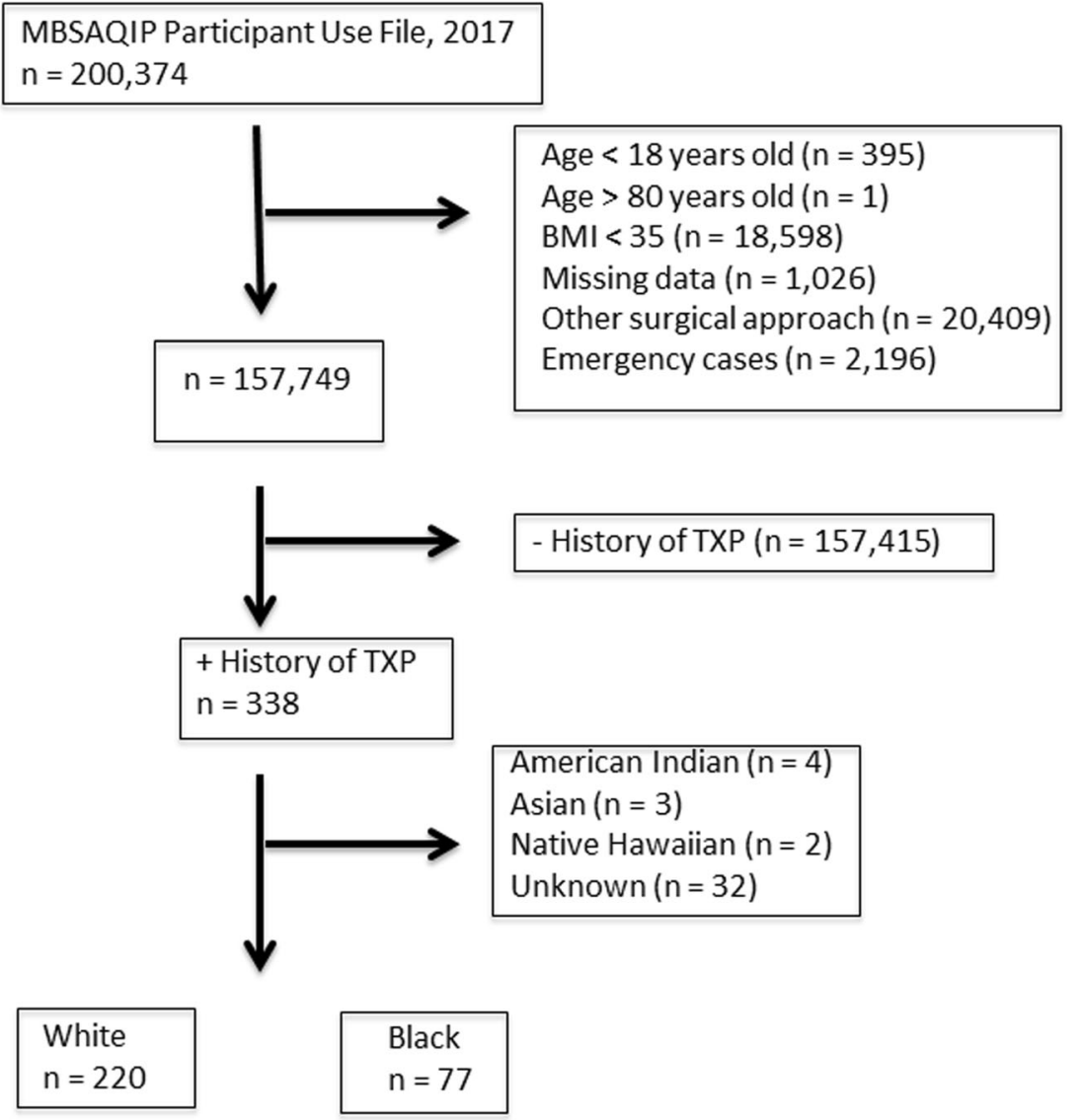

for continuous variables. Continuous data is expressed as median and interquartile range (IQR) and categorical data is expressed as frequency and percentage. All statistical analyses were performed with SPSS version 26 (IBM Corporation, Armonk, NY) and SAS version 9.4 (SAS Institute, Cary, NC). A $p$ value of $<0.05$ was considered statistically significant.

\section{Results}

\section{Patient Demographics}

Of the 200,374 cases in the 2017 MBSAQIP database, $0.3 \%$ $(n=614)$ had a PSOT. Following exclusions, 335 patients with a prior solid organ transplant were analyzed, including 250 white and 85 black patients. Thirty-four percent $(n=208)$ of the transplant cohort was excluded for CPT codes associated with procedures other than RYBG and SG. Characteristics of the unmatched racial cohorts are detailed in Table 1. Patient demographics (age, gender, and body mass index), operative characteristics (procedure type (RYGB vs. SG), surgical approach (conventional laparoscopy vs. roboticassisted)), and most preoperative comorbidities were similar between racial cohorts. Black patients had significantly higher rates of hypertension ( $77 \%$ vs. $64 \%, p=0.03)$, chronic steroid use $(52 \%$ vs. $38 \%, p=0.03)$, and dialysis-dependent chronic kidney disease $(15 \%$ vs. $6.8 \%, p=0.02)$.

\section{Outcomes Following Unmatched Analysis}

Primary and secondary outcome measures were compared in the unmatched racial cohorts and are detailed in Table 2. Overall mortality and morbidity were $0.3 \%$ and $12 \%$, respectively. All-cause mortality ( $0 \%$ vs. $0.4 \%, p=1.0)$ and morbidity $(17 \%$ vs. $10 \%, p=0.1)$ were similar between black and white patients. Bariatric-related morbidity was twofold higher in black patients (14\% vs. $7.2 \%)$ and trended towards significance $(p=0.05)$. In unmatched cohort analysis, black patients had higher rates of postoperative pneumonia $(p=0.02)$, acute renal failure $(p=0.02)$, aggregate pulmonary complications $(p=0.02)$, and emergency (ED) department visit $(p=0.03)$. All other outcome measures were similar (Table 2).

\section{Outcomes Following Propensity Score Matching and Multivariate Logistic Regression Analysis}

One-to-one propensity score analysis compared 82 black and 82 white MBS patients with PSOT that were statistically similar $(p>0.1)$ at baseline (Supplementary Table 1). 
Table 1 Unmatched patient characteristics

\begin{tabular}{|c|c|c|c|c|c|}
\hline & $\begin{array}{l}\text { Total } \\
(n=335)\end{array}$ & $\begin{array}{l}\text { White } \\
(n=250)\end{array}$ & $\begin{array}{l}\text { Black } \\
(n=85)\end{array}$ & $p$ Value & Effect size \\
\hline \multicolumn{6}{|l|}{ Continuous variables, median } \\
\hline \multirow[t]{2}{*}{ Age, years } & $49(41-58)$ & $49(41-59)$ & $48(41-57)$ & 0.317 & 0.17 \\
\hline & $42.1(38.5-$ & $41.6(38.5-$ & 42.9 (38.6- & & \\
\hline BMI, kg/m2 & 46.2) & 46.2) & 46.2) & 0.467 & 0.14 \\
\hline \multicolumn{6}{|l|}{ Categorical variables, $n,(\%)$} \\
\hline Gender, female & $237(71)$ & $173(69)$ & $64(75)$ & 0.286 & 1.26 \\
\hline Ethnicity (Hispanic) & $46(14)$ & $45(18)$ & $1(1.2)$ & $<0.001$ & 13 \\
\hline ASA class $>3$ & $297(89)$ & $218(87)$ & $79(93)$ & 0.149 & 1.93 \\
\hline Operation type & & & & 0.141 & 1.42 \\
\hline Sleeve & $252(75)$ & $183(73)$ & $69(81)$ & & \\
\hline Gastric bypass & $83(25)$ & $67(27)$ & $16(19)$ & & \\
\hline Surgical approach & & & & 0.454 & 0.92 \\
\hline Laparoscopic & $309(92)$ & $229(92)$ & $80(94)$ & & \\
\hline Robotic & $26(8)$ & $21(8)$ & $5(6)$ & & \\
\hline History of myocardial infarction & $9(2.7)$ & $8(3.2)$ & $1(1.2)$ & 0.458 & 2.32 \\
\hline History of PCI & $16(4.8)$ & $13(5.2)$ & $3(3.5)$ & 0.533 & 1.37 \\
\hline History of Cardiac Surgery & $23(6.9)$ & $20(8.0)$ & $3(3.5)$ & 0.159 & 2.02 \\
\hline Hypertension & $225(67)$ & $160(64)$ & $65(77)$ & 0.034 & 1.83 \\
\hline Diabetes mellitus & $117(35)$ & $92(37)$ & $25(29)$ & 0.217 & 0.92 \\
\hline Hyperlipidemia & $135(40)$ & $103(41)$ & $32(38)$ & 0.564 & 1.12 \\
\hline Gastroesophageal reflux disease & $143(43)$ & $112(45)$ & $31(37)$ & 0.180 & 1.30 \\
\hline COPD & $4(1.2)$ & $4(1.6)$ & $0(0)$ & 0.241 & 0.74 \\
\hline Obstructive sleep apnea & $118(35)$ & $95(38)$ & $23(27)$ & 0.068 & 0.89 \\
\hline Oxygen dependent & $4(1.2)$ & $3(1.2)$ & $1(1.2)$ & 0.986 & 1.02 \\
\hline Smoker & $11(3.3)$ & $8(3.2)$ & $3(3.5)$ & 0.883 & 1.11 \\
\hline Renal insufficiency & $47(14)$ & $30(12)$ & $17(20)$ & 0.067 & 1.83 \\
\hline Dialysis & $30(9)$ & $17(6.8)$ & $13(15)$ & 0.018 & 2.50 \\
\hline DVT & $14(4.2)$ & $9(3.6)$ & $5(5.9)$ & 0.364 & 1.67 \\
\hline Pulmonary embolism & $11(3.3)$ & $6(2.4)$ & $5(5.9)$ & 0.120 & 2.54 \\
\hline IVC filter & $6(1.8)$ & $3(1.2)$ & $3(3.5)$ & 0.162 & 1.50 \\
\hline Anticoagulation & $24(7.2)$ & $15(6)$ & $9(11)$ & 0.156 & 1.72 \\
\hline Chronic steroids & $140(42)$ & $96(38)$ & $44(52)$ & 0.031 & 1.72 \\
\hline Limited ambulation status & $9(2.7)$ & $5(2.0)$ & $4(4.7)$ & 0.239 & 2.42 \\
\hline Independent functional status & $329(98)$ & 247 (99) & $82(97)$ & 0.170 & 2.01 \\
\hline Prior bariatric/foregut surgery & $23(6.9)$ & $19(7.6)$ & $4(4.7)$ & 0.362 & 1.49 \\
\hline
\end{tabular}

$I Q R$ interquartile range, $B M I$ body mass index $\left(\mathrm{kg} / \mathrm{m}^{2}\right), A S A$ American society of anesthesiologists, $P C I$ percutaneous coronary intervention, $C O P D$ chronic obstructive pulmonary disease, $D V T$ deep vein thrombosis, $I V C$ inferior vena cava, Bold statistically significant, $p<0.05$

Perioperative outcomes after propensity score matching are outlined in Table 3. There was no mortality in either cohort. Overall (17\% vs. $10 \%, p=0.2)$ and bariatric-related morbidity ( $15 \%$ vs. $8.5 \%, p=0.2$ ) were statistically similar between black and white patients. All other outcome measures were also similar between racial cohorts. Multivariate regression analyses were performed to identify independent predictors of overall and bariatric-related morbidity as shown in Table 4. Black race was not found to be an independent predictor of overall morbidity ( $\mathrm{OR} 1.6, p=0.2)$ or bariatricrelated morbidity (OR 1.78, $\mathrm{p}=0.2)$. Both overall and bariatric-related morbidities were independently associated with chronic steroid use.

\section{Procedure-Specific Analysis}

Subgroup analyses of SG $(n=252)$ and RYGB $(n=83)$ cohorts were performed. Descriptive statistics of these 
Table 2 Unmatched perioperative outcomes

$\begin{array}{llll}\begin{array}{l}\text { Total } \\ (n=335)\end{array} & \begin{array}{l}\text { White } \\ (n=250)\end{array} & \begin{array}{l}\text { Black } \\ (n=85)\end{array} & p \text { Value }\end{array}$

Hospital outcomes, median (IQR)

Operative time, minutes

Length of stay, days

$97(64-136) \quad 100(63-135)$

$94(64-138)$

0.853

30-day adverse outcomes and

$2(1-2)$

$2(1-2)$

$2(1-3)$

0.091

postoperative complications, $n,(\%)$

Overall mortality

$1(0.3)$

$1(0.4)$

$0(0)$

1.000

Bariatric-related mortality

$1(0.3)$

$1(0.4)$

$0(0)$

1.000

Overall morbidity

$39(12)$

$25(10)$

14 (17)

0.108

Bariatric-related morbidity

$30(9)$

$18(7.2)$

1 (14)

0.054

Reoperation

4 (1.6)

0.651

Reoperation related

4 (1.6)

2 (2.4)

0.651

Readmission

$6(1.8)$

$2(2.4)$

0.126

Readmission related

$21(8.4)$

12 (14)

0.056

Postoperative intervention

$27(8.1)$

$11(13)$

0.183

Postoperative intervention related

9 (2.7)

$5(2.0)$

4 (4.7)

0.183

Unplanned ICU admission

9 (2.7)

$5(2.0)$

4 (4.7)

0.069

Perioperative transfusions

$5(2.0)$

5 (5.9)

0.826

Myocardial infarction

9 (2.7)

$7(2.8)$

$1(0.4)$

2 (2.4)

0.559

Pulmonary embolism

$1(0.4)$

0.422

Pneumonia

$0(0)$

$4(1.6)$

$1(0.4)$

$4(1.6)$

$1(0.4)$

4 (1.6)

$0(0)$

$0(0)$

0.015

Superficial SS

$4(1.2)$

$1(1.2)$

0.598

$0(0)$

0.559

Organ space infection

$4(1.2)$

$0(0)$

0.241

Sepsis

$1(0.3)$

$0(0)$

0.559

Progressive renal insufficiency

$4(1.2)$

$0(0)$

0.241

Acute renal failure

2 (0.6)

$0(0)$

0.015

Urinary tract infection

$1(0.4)$

0.559

Clostridium difficile

$1(0.4)$

0.559

Emergency department visits

$1(0.3)$

$0(0)$

$\mathbf{0 . 0 2 6}$

Approach converted

$25(7.5)$

$14(5.6)$

0.449

Aggregate complications, $n$ (\%)

Bleeding

$5(1.5)$

3 (1.2)

11 (13)

Anastomotic leak

$1(0.3)$

$1(0.4)$

0.559

7 (2.1)

$7(2.8)$

0.119

Cardiovascular

$2(0.8)$

0.408

Pulmonary

$0(0)$

$\mathbf{0 . 0 1 5}$

Renal

$2(2.6)$

4 (1.6)

0.651

Venous thromboembolic

$3(1.2)$

0.449

Surgical site infections

$5(1.5)$

$10(4.0)$

0.061

$I Q R$ interquartile range, ICU intensive care unit, SSI surgical site infection, Bold statistically significant, $p<0.05$

procedure-specific analyses are detailed in Supplementary Table 2. For SG cases, female sex $(p=0.04)$ was more prevalent in black patients and preoperative obstructive sleep apnea $(p=0.03)$ was more prevalent in white patients. For RYGB cases, preoperative BMI $(p=0.03)$ and renal insufficiency $(19 \%$ vs. $3 \%, p=0.05)$ were higher in black patients. For both SG and RYGB, all other patient demographics and preoperative comorbidities were similar between racial cohorts. Outcomes of these unmatched procedure-specific subgroup analyses are reported in Supplementary Table 3. Mortality was $0-0.5 \%(p>0.5)$. There were no procedurespecific statistically significant racial differences in overall or bariatric-related morbidity; however, morbidity was higher in black patients for both SG and RYGB cases. For SG, there 
Table 3 Propensity score-matched perioperative outcomes

\begin{tabular}{|c|c|c|c|c|}
\hline & $\begin{array}{l}\text { Total } \\
(n=164)\end{array}$ & $\begin{array}{l}\text { White } \\
(n=82)\end{array}$ & $\begin{array}{l}\text { Black } \\
(n=82)\end{array}$ & $p$ Value \\
\hline \multicolumn{5}{|l|}{ Hospital outcomes, median (IQR) } \\
\hline Operative time, minutes & $95(63-139)$ & $95(57-151)$ & $95(65-137)$ & 0.732 \\
\hline Length of stay, days & $2(1-3)$ & $2(1-2)$ & $2(1-3)$ & 0.649 \\
\hline \multicolumn{5}{|l|}{$\begin{array}{l}\text { 30-day adverse outcomes and } \\
\text { postoperative complications } n,(\%)\end{array}$} \\
\hline Overall morbidity & $22(13)$ & $8(10)$ & $14(17)$ & 0.169 \\
\hline Bariatric-related morbidity & $19(12)$ & $7(8.5)$ & $12(15)$ & 0.222 \\
\hline Reoperation & $3(1.8)$ & $1(1.2)$ & $2(2.4)$ & 0.560 \\
\hline Reoperation related & $3(1.8)$ & $1(1.2)$ & $2(2.4)$ & 0.560 \\
\hline Readmission & $19(12)$ & $7(8.5)$ & $12(15)$ & 0.222 \\
\hline Readmission related & $18(11)$ & $7(8.5)$ & $11(13)$ & 0.318 \\
\hline Postoperative intervention & $5(3.0)$ & $1(1.2)$ & $4(4.9)$ & 0.173 \\
\hline Postoperative intervention related & $5(3.0)$ & $1(1.2)$ & $4(4.9)$ & 0.173 \\
\hline ICU admission & $8(4.9)$ & $3(3.7)$ & $5(6.1)$ & 0.468 \\
\hline Perioperative transfusions & $6(3.7)$ & $4(4.9)$ & $2(2.4)$ & 0.405 \\
\hline Myocardial infarction & $1(0.6)$ & $1(1.2)$ & $0(0)$ & 0.316 \\
\hline Pulmonary embolism & $1(0.6)$ & $0(0)$ & $1(1.2)$ & 0.316 \\
\hline Pneumonia & $2(1.2)$ & $0(0)$ & $2(2.4)$ & 0.155 \\
\hline Deep incisional SSI & $1(0.6)$ & $1(1.2)$ & $0(0)$ & 0.316 \\
\hline Organ space infection & $1(0.6)$ & $1(1.2)$ & $0(0)$ & 0.316 \\
\hline Progressive renal insufficiency & $3(1.8)$ & $3(3.7)$ & $0(0)$ & 0.080 \\
\hline Acute renal failure & $2(1.2)$ & $0(0)$ & $2(2.4)$ & 0.155 \\
\hline Urinary tract infection & $1(0.6)$ & $1(1.2)$ & $0(0)$ & 0.316 \\
\hline Clostridium difficile & $1(0.6)$ & $1(1.2)$ & $0(0)$ & 0.316 \\
\hline Emergency department visits & $14(8.5)$ & $4(4.9)$ & $10(12)$ & 0.094 \\
\hline Approach converted & $2(1.2)$ & $1(1.2)$ & $1(1.2)$ & 1.000 \\
\hline \multicolumn{5}{|l|}{ Aggregate complications, $n(\%)$} \\
\hline Bleeding & $0(0)$ & $0(0)$ & $0(0)$ & - \\
\hline Anastomotic leak & $4(2.4)$ & $4(4.9)$ & $0(0)$ & 0.120 \\
\hline Cardiovascular & $1(0.6)$ & $1(1.2)$ & $0(0)$ & 0.316 \\
\hline Pulmonary & $2(1.2)$ & $0(0)$ & $2(2.4)$ & 0.155 \\
\hline Renal & $5(3.0)$ & $3(3.7)$ & $2(2.4)$ & 0.650 \\
\hline Venous thromboembolic & $4(2.4)$ & $2(2.4)$ & $2(2.4)$ & 1.000 \\
\hline Surgical site infections & $3(1.8)$ & $3(3.7)$ & $0(0)$ & 0.080 \\
\hline
\end{tabular}

$I Q R$ interquartile range, $I C U$ intensive care unit, $S S I$ surgical site infection

was a higher rate of aggregate pulmonary complications $(2.9 \%$ vs. $0 \%, p=0.07)$ and $\mathrm{ED}$ visits $(10 \%$ s. $3.8 \%, p=$ $0.05)$, though it did not reach statistical significance. For RYGB cases, there was an 8.6-fold higher likelihood of ICU admission for black patients; however, the difference was not statistically significant $(p=0.09)$. Procedure-specific multivariate regression analyses were performed and results are detailed in Table 5. For SG cases, black race was associated with a higher likelihood of overall (OR 2.3, CI 0.92-5.73, $p=$ 0.07 ) and bariatric-related morbidity (OR 2.78, CI 0.98-7.89, $p=0.06$ ), but not significantly so. Obstructive sleep apnea also independently correlated with bariatric-related morbidity (OR 2.95, CI 1.06-8.24, $p=0.04$ ). While overall (OR 2.08) and bariatric related (OR 2.52) morbidity were also higher in black patients after RYGB, the differences were not significant (Table 6).

\section{Discussion}

This is the first and largest study reporting on disparity in outcomes following metabolic and bariatric surgery in racial 
Table 4 Multivariable regression for risk factors associated with overall and bariatric-related morbidity

\begin{tabular}{llll}
\hline & Odds ratio & $95 \%$ CI & $p$ Value \\
\hline Overall morbidity & & & \\
Race (Black vs. White) & 1.60 & $0.76-3.82$ & 0.218 \\
Preoperative steroid use & 2.40 & $1.17-4.92$ & $\mathbf{0 . 0 1 7}$ \\
Preoperative dialysis & 1.04 & $0.33-3.26$ & 0.941 \\
Hispanic & 1.00 & $0.35-2.83$ & 0.993 \\
Hypertension & 0.95 & $0.43-2.07$ & 0.890 \\
Bariatric-related morbidity & & & \\
Race (Black vs. White) & 1.78 & $0.79-4.02$ & 0.168 \\
Preoperative steroid use & 2.25 & $1.05-4.84$ & $\mathbf{0 . 0 3 8}$ \\
Preoperative dialysis & 1.48 & $0.47-4.71$ & 0.506 \\
Hypertension & 1.32 & $0.53-3.32$ & 0.556 \\
Hispanic & 0.81 & $0.22-2.93$ & 0.747 \\
\hline
\end{tabular}

Bold statistically significant, $p<0.05$

cohorts with a history of prior solid organ transplantation. In this analysis, we have reinforced the safety of MBS in transplant patients, showing a low rate of overall mortality $(0.3 \%)$, overall morbidity $(12 \%)$, bariatric-related mortality $(0.3 \%)$, and bariatric-related morbidity (9\%). This is consistent with the published literature on perioperative outcomes following MBS. The systematic review of randomized and observational studies $(n=161,756)$ by Chang et al. [3] reported mortality and morbidity rates of $0.22-0.35 \%$ and $9.8 \%$, respectively, following metabolic and bariatric surgery. Following propensity score-matched analyses in our study, there were no statistically significant differences in hospital outcomes, 30-day adverse outcomes, and postoperative or aggregate complications between racial cohorts. Nonetheless, the rates of readmission (1.8-fold), reoperation (2-fold), reintervention (4-

Table 5 Multivariable regression for risk factors associated with overall and bariatric-related morbidity in SG patients

\begin{tabular}{llll}
\hline & Odds ratio & $95 \%$ CI & $p$ Value \\
\hline Overall morbidity & & & \\
Race (Black vs. White) & 2.30 & $0.92-5.73$ & 0.074 \\
Obstructive sleep apnea & 2.39 & $0.99-5.77$ & 0.052 \\
Hispanic & 1.16 & $0.31-4.36$ & 0.825 \\
Male (vs. Female) & 1.10 & $0.44-2.75$ & 0.836 \\
Bariatric-related morbidity & & & \\
Race (Black vs. White) & 2.78 & $0.98-7.89$ & 0.055 \\
Obstructive sleep apnea & 2.95 & $1.06-8.24$ & $\mathbf{0 . 0 3 9}$ \\
Hispanic & 1.11 & $0.23-5.44$ & 0.898 \\
Male (vs. Female) & 0.94 & $0.32-2.76$ & 0.906 \\
\hline
\end{tabular}

$S G$ sleeve gastrectomy, Bold statistically significant, $p<0.05$
Table 6 Multivariable regression for risk factors associated with overall and bariatric-related morbidity in RYGB patients

\begin{tabular}{llll}
\hline & Odds ratio & $95 \%$ CI & $p$ Value \\
\hline Overall morbidity & & & \\
Race (Black vs. White) & 2.08 & $0.48-9.09$ & 0.330 \\
Hispanic & 0.99 & $0.18-5.45$ & 0.993 \\
Preoperative renal insufficiency & 0.88 & $0.08-9.61$ & 0.917 \\
BMI & 0.65 & $0.11-3.72$ & 0.626 \\
Bariatric-related morbidity & & & \\
Race (Black vs. White) & 2.52 & $0.55-11.5$ & 0.233 \\
Preoperative renal insufficiency & 1.01 & $0.09-11.4$ & 0.992 \\
Hispanic & 0.55 & $0.06-5.01$ & 0.593 \\
BMI & 0.50 & $0.08-2.98$ & 0.449 \\
Race (Black vs. White) & 2.52 & $0.55-11.5$ & 0.233 \\
\hline$R Y G B$ Roux-en-Y gastric bypass, $B M I$ body mass index, Bold statistically \\
significant, $p<0.05$
\end{tabular}

fold), and emergency department (ED) visits (2.4-fold) were higher in black patients. On multivariate regression analysis, black race was not found to be an independent predictor of allcause or bariatric-related morbidity. While race was not an independent predictor of outcomes in this cohort of MBS patients with prior solid organ transplantation, chronic steroid use was.

The nonsignificant differences in outcomes noted between black and white patients in this study are consistent with prior studies reporting a lack of significant correlation between race and MBS outcomes [21-23]. However, the nonsignificantly higher rates of some adverse outcomes (reoperation, readmission, reintervention) in black patients in our study are concerning and need further exploration with larger patient cohorts. It is possible that these differences represent racial disparity in outcomes, but given the small sample sizes, the study had inadequate power to show a statistically significant difference.

Upon multivariable regression analysis of SG and RYGB cases independently, black race was associated with a high odds ratio $(\mathrm{OR}>2)$ for both overall and bariatric-related morbidity, more so with SG. However, these differences did not reach statistical significance. In our study, we also notice a trend toward a higher rate of ED visits (2.6-fold) in black SG patients. The reasons for this finding remain unclear and could not be elucidated from the database used for our study. In the published literature, the most common reported reasons for ED visits including abdominal pain, nausea/vomiting, dehydration, wound concerns, and compliance issues [26-29]. In a recent prospective cohort study of patients in the Michigan Bariatric Surgery Collaborative (MBSC) database, Stevens et al. [26] found that most ED presenting symptoms following bariatric surgery were of low acuity and non-life- 
threatening. Some studies have highlighted educational level as a possible contributor to poor compliance, resulting in higher readmission rates. Mahoney et al [30] evaluated grade level education (survey) and health literacy using the Rapid Estimate of Adult Literacy in Medicine-Short Form (REALM-SF) health literacy test and found that educational level less than or equal to 12th grade was associated with a threefold higher rate of ED visits and readmissions following bariatric surgery. No correlation was found between health literacy and ED visits. Others have reported patient demographics, such as younger age and female sex as potential predictors of ED visits [28]. Given the higher associated healthcare cost from ED visits and readmissions, there is increasing interest to improve this MBS quality metric. Further studies to differentiate reasons for ED visits and readmissions between racial cohorts and high-risk patient cohorts (e.g., prior transplantation) may further our understanding of this quality metric and highlight opportunities for improvement, efficiency, and cost-savings. In contrast to numerous studies showing a higher rate of adverse outcomes following RYGB compared with $\mathrm{SG}$, in our procedure-specific analyses of racial bariatric surgery cohorts with prior solid organ transplantation, there were no significant differences in outcomes between RYGB and SG cases.

While our study demonstrated that outcomes in this cohort of MBS patients are not mediated by race, there are several study limitations to consider. First, this is a retrospective analysis that is subject to the inherent biases of such an analysis. Second, a significant portion of transplant cases were excluded, primarily because of CPT codes not specific to SG or RYGB. Even though this represents the largest study in this cohort of patients, generalizability is limited by the overall small sample size analyzed. In addition, several granular data points are not included in the database and therefore could not be accounted for in our analysis. Such data points include the type of solid organ transplantation performed, timeframe from transplantation to bariatric surgery, and the immunosuppressive regimen of each patient. The timeframe between organ transplantation and metabolic and bariatric surgery may impact intraoperative findings, operative course, and ultimately perioperative outcomes. The inability to account for these confounders may have an impact on our findings.

\section{Conclusion}

Metabolic and bariatric surgery in racial cohorts of post-solid organ transplantation patients is safe, with very low rates of morbidity and mortality. While some adverse outcomes were higher in black patients, race was not found to be an independent predictor of adverse outcomes following SG or RYGB in patients with prior solid organ transplantation. However, there were trends toward higher rates of adverse outcomes, including overall and bariatric-related morbidity, in both propensity score-matched and multivariate regression analyses. Given the sample size limitation of our study, additional analyses of larger cohorts are needed to further explore the impact of race on outcomes in bariatric surgery patients with prior organ transplantation.

\section{Compliance with Ethical Standards}

Conflict of Interest The authors declare that they have no conflict of interest.

Ethical Approval Statement This is a retrospective study of nationally available de-identified data. For this type of study formal consent is not required.

Informed Consent Statement Informed consent does not apply.

\section{Appendix}

Table 7 Composite complication methodology

\begin{tabular}{ll}
\hline Aggregate variable & Composite variables \\
\hline Leak & Reoperation with suspected reason: leak \\
& Readmission with suspected reason: leak \\
& Intervention with suspected reason: leak \\
& Drain present over 30 days \\
& Complication: organ space SSI \\
& Reoperation with suspected reason: bleeding \\
Bleeding & Readmission with suspected reason: bleeding \\
& Intervention with suspected reason: bleeding
\end{tabular}


Table 7 (continued)

Aggregate variable

Cardiac/CVA

Pulmonary

Renal

DVT or PE

Wound infection

Other infection

Overall morbidity

Aggregate-related reoperation

Aggregate-related readmission

Aggregate-related intervention

Bariatric surgery-related morbidity
Composite variables

Reoperation with suspected reason: cardiac NOS, CVA, or MI

Readmission with suspected reason: cardiac NOS, CVA, or MI

Intervention with suspected reason: cardiac NOS, CVA, or MI

Complication of CVA

Complication of MI

Reoperation with suspected reason: shortness of breath, pneumonia, or other respiratory failure Readmission with suspected reason: shortness of breath, pneumonia, or other respiratory failure Intervention with suspected reason: shortness of breath, pneumonia, or other respiratory failure Complication: on ventilator $>48 \mathrm{~h}$

Complication: unplanned intubation

Complication: pneumonia

Reoperation with suspected reason: renal insufficiency

Readmission with suspected reason: renal insufficiency

Intervention with suspected reason: renal insufficiency

Complication: progressive renal insufficiency

Complication: acute renal failure

Reoperation with suspected reason: vein thrombosis requiring therapy or pulmonary embolism

Readmission with suspected reason: vein thrombosis requiring therapy or pulmonary embolism Intervention with suspected reason: vein thrombosis requiring therapy or pulmonary embolism Complication: vein thrombosis requiring therapy

Complication: pulmonary embolism

Complication: anticoagulation initiated of presumed/confirmed vein thrombosis/PE

Reoperation with suspected reason: wound infection or other abdominal sepsis

Readmission with suspected reason: wound infection or other abdominal sepsis

Intervention with suspected reason: wound infection or other abdominal sepsis

Complication: post-op superficial incisional SSI occurrence

Complication: post-op deep incisional SSI occurrence

Reoperation with suspected reason: infection/fever

Readmission with suspected reason: infection/fever,

Intervention with suspected reason: infection/fever

Complication: post-op sepsis occurrence

Complication: post-op septic shock occurrence

Complication: post-op pneumonia occurrence

Complication: post-op urinary tract infection occurrence

Mortality within 30 days

Need for intervention within 30 days

Need for readmission within 30 days

Need for reoperation within 30 days

Unplanned ICU transfer within 30 days

Any reoperation designated as related to metabolic/bariatric by variable REOP_RELATED_ BAR1. To REOP_RELATED_BAR.13

Any readmission designated as related to metabolic/bariatric by variable READ_ RELATED BAR1. To READ RELATED BAR.11

Any intervention designated as related to metabolic/bariatric by variable INVT RELATED_BAR1. To INTV_RELATED_BAR.5

Death related to bariatric surgery

Aggregate reoperation related to metabolic/bariatric surgery

Aggregate readmission related to metabolic/bariatric surgery

Aggregate intervention related to metabolic/bariatric surgery 


\section{References}

1. Hales C, Fryar CD, Carroll MD, et al. Differences in obesity prevalence by demographic characteristics and urbanization level among adults in the Unites States, 2013-2016. JAMA. 2018;319(23):2319-429.

2. Flegal KM, Kruszon-Moran D, Carroll MD, et al. Trends in obesity among adults in the United States, 2005 to 2014. JAMA. 2016;315(21):2284-91.

3. Chang SH, Stoll CRT, Song J, et al. The effectiveness and risks of bariatric surgery: an updated systematic review and meta-analysis, 2003-2012. JAMA Surg. 2014;149(3):275-87.

4. Yu J, Zhou X, Li L, et al. The long-term effects of bariatric surgery for type 2 diabetes: systematic review and meta-analysis of randomized and non-randomized evidence. Obes Surg. 2015;25:143-58.

5. Schauer PR, Bhatt DL, Kirwan JP, et al. Bariatric surgery versus intensive medical therapy for diabetes-5-year outcome. NEJM. 2017;376(7):641-51.

6. Kashyap SR, Bhatt DL, Wolski K, et al. Metabolic effects of bariatric surgery in patients with moderate obesity and type 2 diabetes: analysis of a randomized control trial comparing surgery with intensive medical treatment. Diabetes Care. 2013;36(8):2175-82.

7. Christou NV. Impact of obesity and bariatric surgery on survival. World J Surg. 2009;33(10):2022-7.

8. Fouse T, Brethauer S. Resolution of comorbidities and impact on longevity following bariatric and metabolic surgery. Surg Clin North Am. 2016;96(4):717-32.

9. Hachem A, Brennan L. Quality of life outcomes of bariatric surgery: a systematic review. Obes Surg. 2016;26(2):395-409.

10. Mazer LM, Azagury DE, Morton JM. Quality of life after bariatric surgery. Curr Obes Rep. 2017;6(2):204-10.

11. Yemini R, Nesher E, Winkler J, et al. Bariatric surgery in solid organ transplant patients: long-term follow-up results of outcome, safety, and effect on immunosuppression. Am J Transplant. 2018;18(11):2772-80.

12. Khoraki J, Katz MG, Funk LM, et al. Feasibility and outcomes of laparoscopic sleeve gastrectomy after solid organ transplantation. Surg Obes Relat Dis. 2016;12(1):75-83.

13. Lin MY, Tavakol MM, Sarin A, et al. Laparoscopic sleeve gastrectomy is safe and efficacious for pretransplant candidates. Surg Obes Relat Dis. 2013;9(5):653-8.

14. Bennett WM, McEvoy KM, Henell KR, et al. Kidney transplantation in the morbidly obese: complicated but still better than dialysis. Clin Transpl. 2011;25(3):401-5.

15. Elli EF, Gonzalez-Heredia R, Sanchez-Johnsen L, et al. Sleeve gastrectomy surgery in obese patients post-organ transplantation. Surg Obes Relat Dis. 2016;12(3):528-34.
16. Kienzl-Wagner K, Weissenbacher A, Gehwolf $\mathrm{P}$, et al. Laparoscopic sleeve gastrectomy: gateway to kidney transplantation. Surg Obes Relat Dis. 2017;13(6):909-15.

17. Wang Y, Beydoun MA, Liang L, et al. Will all Americans become overweight or obese? Obesity. 2008;16:2323-30.

18. Zhang, Rodriguez-Monguio. Racial disparities in the risk of developing obesity-related diseases: a cross-sectional study. Ethn Dis. 2012;22(3):308-16

19. Cohen et al. A pooled analysis of body mass index and mortality among African Americans. PLoS One. 2014;9(11):e111980.

20. Chang SH, Yu YC, Carlsson NP, et al. Racial disparity in life expectancies and life years lost associated with multiple obesityrelated conditions. Obesity. 2017;25(5):950-7.

21. Elli FE et al. Bariatric surgery outcomes in ethnic minorities. Surgery. 2016;160:805-12.

22. Flum DR et al. Longitudinal assessment of bariatric surgery (LABS) consortium, perioperative safety in the longitudinal assessment of bariatric surgery. NEJM. 2009;361(5):445-54.

23. Ng J, Seip R, Stone A, et al. Ethnic variation in weight loss, but no co-morbidity remission, after laparoscopic gastric banding and Roux-en-Y gastric bypass. Surg Obes Relat Dis. 2015;11:94-100.

24. Turner PL, Oyetunji TA, Gantt G, et al. Demographically associated variations in outcomes after bariatric surgery. Am J Surg. 2011;201:475-80.

25. Weller WE, Rosati C, Hannan E. Predictors of in-hospital postoperative complications among adults undergoing bariatric surgery in New York State, 2003. Obes Surg. 2006;16:702-8.

26. Stevens H, Wells E, Ross R, et al. Patient perspectives on emergency department self-referral after bariatric surgery. Surg Obes Relat Dis. 2018;14(5):674-81.

27. Chen J, Mackenzie J, Zhai Y, et al. Preventing returns to the emergency department following bariatric surgery. Obes Surg. 2017;27(8):1986-92.

28. Macht R, George J, Ameli O, et al. Factors associated with bariatric postoperative emergency department visits. Surg Obes Relat Dis. 2016;12(10):1826-31.

29. Altieri MS, Yang J, Groves D, et al. Sleeve Gastrectomy: the first 3 years: evaluation of emergency department visits, readmissions, and reoperations for 14,080 patients in New York State. Surg Endosc. 2018;32(3):1209-14.

30. Mahoney ST, Tawfik-Sexton D, Strassle PD, et al. Effects of education and health literacy on postoperative hospital visits in bariatric surgery. J Laparoendosc Adv Surg Tech A. 2018;28(9):1100-4.

Publisher's Note Springer Nature remains neutral with regard to jurisdictional claims in published maps and institutional affiliations. 